\title{
Computational and experimental analysis of flow structures induced by a plasma actuator with burst modulations in quiescent air
}

\author{
Hikaru AONO*, Satoshi SEKIMOTO**, Makoto SATO*, Aiko YAKENO*, Taku NONOMURA* and \\ Kozo FUJII* \\ * Japan Aerospace Exploration Agency \\ 3-1-1 Yoshinodai, Chuo-ku, Sagamihara-shi, Kanagawa 252-5210, Japan \\ E-mail: aono@rs.tus.ac.jp \\ ** Department of Aeronautics and Astronautics, University of Tokyo \\ 3-1-1 Yoshinodai, Chuo-ku, Sagamihara-shi, Kanagawa 252-5210, Japan
}

Received 11 April 2015

\begin{abstract}
Characteristics of flow fields produced by a dielectric barrier discharge plasma actuator in quiescent air are numerically investigated. A time-dependent localized body-force distribution is utilized to mimic the effect of the plasma actuator with modulated bursts. The computed time-averaged and instantaneous flow fields are compared with the experimental results by using high-speed schlieren photography and particle image velocimetry. The computed flow fields are in good agreement with the experimental results when the nondimensional parameter $\left(D_{c}\right)$ is within the appropriate range. With an appropriate choice of $D_{c}$, the location and size of the induced flow structures, computed with respect to the maximum flow velocity parallel to the wall, are quantitatively in agreement with the experimental results. Also considered are the effects of the burst frequency (non-dimensionalized by the chord length and the free-stream velocity of assumed separated flow control experiment) on the induced flow. The results show that changes in the burst frequency cause insignificant changes in the magnitude of the time-averaged flow parallel to the wall, but they cause significant fluctuations in the amplitude and power spectral densities of that flow.
\end{abstract}

Key words : Computational fluid dynamics (CFD), Experimental fluid dynamics (EFD), Control of flow, Dielectric barrier discharge plasma actuator, Induced flow

\section{Introduction}

In order to improve the efficiency and performance of many kinds of fluid machinery, active flow control using dielectric barrier discharge plasma actuators ("plasma actuators") has been actively studied (e.g., Moreau, 2007, Corke, et al., 2009, Cho and Shyy, 2010, Wang, et al., 2013). Plasma actuators have zero net mass flow, unlike, for example, the steady blowing of a jet (Cattefesta and Sheplak, 2011). A schematic diagram of a typical single plasma actuator is shown in Fig. 1; it consists of two thin dielectric electrodes with a dielectric material sandwiched between them. When an alternating-current (AC) voltage of the order of $10^{3}$ volts and with high driving frequencies (i.e., $O\left(10^{3}-10^{4}\right)$ ) is applied between the two electrodes, an atmospheric-pressure nonequilibrium plasma is created, and this induces a wall-jet-like flow above the dielectric (Roth, Sherman, and Wilkinson, 1998, 2000). This induced flow plays a role in separated flow control.

In the past decade, experimental studies have elucidated the relationship between the velocity of the induced wall jet and the parameters of the plasma actuators; this includes both the operational parameters, such as input voltage, frequency, and waveform, and the geometric parameters, such as electrode height, length, and gap (see the excellent review provided by Roth and Dai, 2006, Forte, et al., 2007, Corke, et al., 2009, Wang, et al., 2013, Benard and Moreau, 2014). This relationship is an important aspect of separated flow control. It has been demonstrated that: 1) when the electrodes are 
geometrically optimized, the time-averaged maximum velocity of a wall jet can be up to $7 \mathrm{~m} / \mathrm{s}$; 2) a thick dielectric material enhances the body force; and 3) increased voltages and frequencies increase the velocity of the induced flow.

It has been reported that modulating the input signal (burst input) of the plasma actuator can be used to control the separated flow. However, the characteristics of the resulting flow structures are not yet understood. Recently, Benard and Moreau (2010) considered the wall jet produced by a single asymmetric plasma actuator acting on different modulated waveforms. They considered a peak-to-peak applied voltage $\left(V_{p p}\right)$ of $36,000 \mathrm{~V}$ and a base sinusoidal frequency $\left(f_{\text {base }}\right)$ of $1,500 \mathrm{~Hz}$, and they considered three different types of modulation: burst modulation (see Fig. 2), superposition, and ring modulation. They used a mono-component laser Doppler velocimeter to measure the time-resolved velocities and power spectral densities (PSDs). Their results showed that burst modulation with a burst ratio $\left(\mathrm{BR}=t_{\text {on }} f_{\text {burst }}\right)$ or duty cycle of $50 \%$ resulted in two frequency peaks, each of which is the result of superimposing numerous harmonics; for this case, the time-averaged velocity was low, and there were rapid fluctuations in the bust frequency $\left(f_{\text {burst }}\right)$, which was low. Kotsonis and Veldhuis (2010) used hot-wire probes to measure the total velocity induced by a single asymmetric plasma actuator with modulated bursts; $V_{p p}$ was $10,000 \mathrm{~V}$, and $f_{\text {base }}$ was $2,000 \mathrm{~Hz}$. Several combinations of $f_{\text {burst }}$ and BR were tested. The results showed that when burst modulation is used, the plasma actuator is capable of inducing in the surrounding air both the mean velocity and the fluctuating components. They found that the mean total velocity and the fluctuating intensity were strongly dependent on $f_{\text {burst }}$ and BR. Similar results for this dependency were reported in the previous study (Benard and Moreau, 2010). These studies clearly have contributed to the ability to perform better separated flow control.

There has been a recent increase in the number of numerical simulations related to the use of plasma actuators for flow control (see Shang and Huang, 2014). These studies can be grouped by how much detail they use to model the physics associated with the plasma actuator. For example, the kinematic approach is very detailed but computationally very expensive, the continuous model approach that solves the drift-diffusion equations (e.g., Yamamoto and Fukagata, 2013, Nishida, Nonomura, and Abe, 2014) is moderately detailed and computationally expensive, and the continuous model approach that introduces an assumption about the Debye length is less detailed but computationally cheap. This suggests that the degree of detail used to model the plasma actuator should be based on the objectives of the study.

Since our objective is to use active flow control to improve the performance of fluid machinery and transport equipment, it is necessary to simulate the unsteady flow and to include the effects of the plasma actuator. Previous computational studies (Fujii, 2014, Sato, et al., 2015) determined that there are three characteristics of the flow that are important for separated flow control; these are the reattachment due to the free-stream momentum inducted by the laminar-to-turbulent transition of the separated shear layer, the large low-pressure region generated by coherent, large-scale vortex convection near the surface, and the direct momentum addition by the plasma actuator to close to the surface; this last has a chordbased Reynolds number that is $O\left(10^{3}-10^{6}\right)$. The first two phenomena naturally vary in time and space, so they have been varied in many flow control simulations (e.g., Asada and Fujii, 2011, Visbal 2010, Gaitonde, et al., 2006, Gross and Fasel, 2010, Riherd and Roy, 2013). In our previous study (Sato, et al., 2015) using a plasma actuator model, we used a continuous approach with the Debye length assumption, and the results were sufficiently accurate to support a discussion of flow control mechanisms and performance.

Several plasma actuator models that are based on the continuous approach have been developed and are often used in flow control simulations. The body force model of Shyy et al. (2002) is one of the best known of these models; their model of the plasma actuator assumes that the electric field is linear in space, the body force acts only in regions in which the plasma is present and while it is being formed, and the force is assumed to be independent of time. In a case study of the laminar boundary layer, their results were consistent with experimental observations. In the Suzen-Huang body force model (Suzen, et al., 2005), it is necessary to solve two equations to obtain the body force fields, prior to performing the flow control simulations; the maximum induced velocity in their model is in reasonable agreement with the results of particle image velocimetry (PIV) experiments. The boundary condition for this model was modified by Ibrahim and Skote (2011), and the modified model gives a more accurate value for the maximum velocity of the wall jet. This model has been used to compute the flow induced by a linear plasma synthetic jet (Ibrahim and Skote, 2012). The body force model of Orlov, et al. (2005) is the most advanced of the models considered here. This model obtains the time-dependent induced flow by solving first-order differential equations with time-dependent boundary conditions. Orlov, et al. (2005) compared the instantaneous flow field with the available experimental data under conditions of quiescent flow.

Recently, time-resolved PIV and Navier-Stokes (NS) computations have been used to estimate the time-dependent body forces (Maden, et al., 2013). Although the plasma actuator is continuously on, the wall-jet profiles predicted by the body force models used in this approach are in reasonable agreement. These results have encouraged us to consider the flow structure induced by burst modulation or a duty cycle. In particular, in the present study, we consider the possibility 


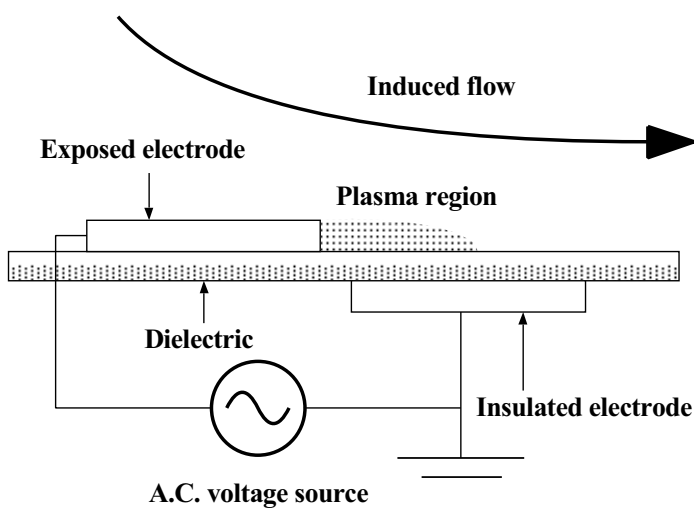

Fig. 1 Configuration of an asymmetric plasma actuator.

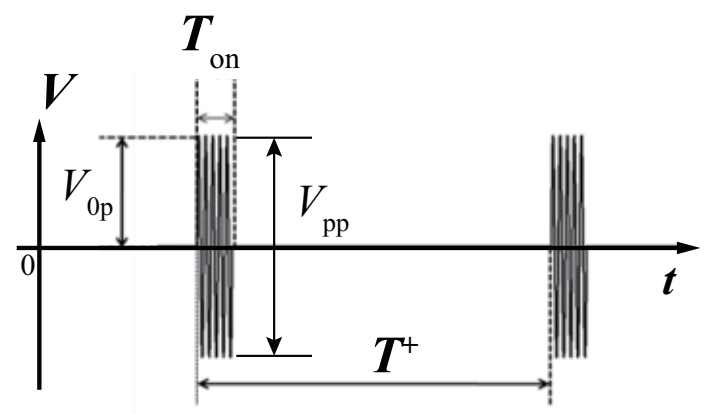

Fig. 2 Schematic diagram of a bursting wave input signal. $V_{p p}$ indicates the peak-to-peak amplitude of the applied voltage.

of predicting the flow induced by a time-varying body force model that is developed based on the Suzen-Huang body force model (Suzen, et al., 2005). We performed a numerical simulation of the induced flow structures in quiescent air, and the computed results were compared both qualitatively and quantitatively with the experimental results. We also consider the way in which the computed flow structure changes due to the burst frequency.

\section{Case descriptions}

\subsection{Computational conditions}

For the initial condition, the flow was assumed to be quiescent around the plasma actuator and the flat plate, and the flow field was assumed to be laminar. The reference length $\left(L_{r e f}\right)$ and velocity $\left(U_{r e f}\right)$ were assumed to be the same as those used in the experimental setup for separated flow control around an airfoil in a low-speed tunnel (Asada, et al., 2009): $L_{r e f}$ is $10 \mathrm{~cm}$, and $U_{r e f}$ is $10 \mathrm{~m} / \mathrm{s}$. The Reynolds number was 63,000, the specific heat ratio $(\gamma)$ was 1.4 , and the Prandtl number $\left(P_{r}\right)$ was 0.72 . The Mach number was set to 0.2 , so the compressibility of the fluid is negligible. For the body force model, we assumed that the base sinusoidal frequency $\left(f_{\text {base }}\right)$ was $10,000 \mathrm{~Hz}$, which corresponds to a nondimensional sinusoidal base frequency $\left(F_{\text {base }}=f_{b a s e} L_{r e f} / U_{\text {ref }}\right)$ of 100 . This was higher than that for the flow fluctuation. We considered three burst frequencies of $100 \mathrm{~Hz}, 500 \mathrm{~Hz}$, and 1,000 Hz, which correspond to nondimensional burst frequencies $\left(F^{+}=f_{\text {burst }} L_{r e f} / U_{\text {ref }}\right)$ of 1,5 , and 10, respectively. When the burst frequency was $100 \mathrm{~Hz}$, we compared the results for five values of $D_{c}$. The burst ratio $\left(\mathrm{BR}=T_{o n} F^{+}\right)$was fixed at $10 \%$. The ranges of $F^{+}$and BR in our study are similar to those used in the airfoil flow control simulations (Sato, et al., 2015) and the experiments (Sekimoto, 2015). The computational cases are listed in Table 1.

Table 1 List of the computational cases

\begin{tabular}{|l|c|c|c|}
\hline Case name & BR [-] & $f_{\text {burst }}[\mathrm{Hz}]$ & $D_{c}[-]$ \\
\hline F1BR10Dc0.0059 & 10 & 100 & 0.0059 \\
\hline F1BR10Dc0.0078 & 10 & 100 & 0.0078 \\
\hline F1BR10Dc0.0117 & 10 & 100 & 0.0117 \\
\hline F1BR10Dc0.0156 & 10 & 100 & 0.0156 \\
\hline F1BR10Dc0.0234 & 10 & 100 & 0.0234 \\
\hline F5BR10Dc0.0117 & 10 & 500 & 0.0117 \\
\hline F5BR10Dc0.0156 & 10 & 500 & 0.0156 \\
\hline F10BR10Dc0.0117 & 10 & 1,000 & 0.0117 \\
\hline F10BR10Dc0.0156 & 10 & 1,000 & 0.0156 \\
\hline
\end{tabular}

Table 2 List of the experimental cases

\begin{tabular}{|l|c|c|c|}
\hline Case name & BR [-] & $f_{\text {burst }}[\mathrm{Hz}]$ & $V_{p p}[\mathrm{kV}]$ \\
\hline F1BR10Vpp7 & 10 & 100 & 7 \\
\hline F5BR10Vpp7 & 10 & 500 & 7 \\
\hline F10BR10Vpp7 & 10 & 1,000 & 7 \\
\hline
\end{tabular}

\subsection{Experimental conditions}

The flow around the plasma actuator and the flat plate in the chambers was very close to quiescent. The peak-to-peak amplitude of the applied voltage $\left(V_{p p}\right)$ was $7 \mathrm{kV}$. The base sinusoidal frequency was set to $10,000 \mathrm{~Hz}$, which corresponds to an $F_{\text {base }}$ of 100 . The burst frequencies were $100 \mathrm{~Hz}, 500 \mathrm{~Hz}$, and $1,000 \mathrm{~Hz}$, which correspond to $F^{+}$values of 1,5 , and 10, respectively. The burst ratio was fixed at $10 \%$. The experimental cases are listed in Table 2. 


\section{Methodologies}

The current study uses a combination of numerical simulations, schlieren photography, and particle image velocimetry in order to compare the flow structures induced by a plasma actuator operated with burst modulations in static air. Below, we give brief descriptions of the numerical and experimental methods.

\subsection{Numerical simulation}

\subsubsection{Governing equations}

The flow field around the plasma actuator and the flat plate is assumed to be described by the NS equations, in which the source terms represent the local forcing of the plasma actuator. In nondimensional form and Cartesian coordinates, the system of governing equations is

$$
\begin{aligned}
& \frac{\partial \rho}{\partial t}+\frac{\partial \rho u_{k}}{\partial x_{k}}=0, \\
& \frac{\partial \rho u_{i}}{\partial t}+\frac{\partial\left(\rho u_{i} u_{k}+p \delta_{i k}\right)}{\partial x_{k}}=\frac{1}{\operatorname{Re}} \frac{\partial \tau_{i k}}{\partial x_{k}}+D_{c} S_{i}, \\
& \frac{\partial e}{\partial t}+\frac{\partial\left((e+p) u_{k}\right)}{\partial x_{k}}=\frac{1}{\operatorname{Re}}\left(\frac{\partial u_{l} \tau_{k l}}{\partial x_{k}}-\frac{1}{(\gamma-1) P_{r} M^{2}} \frac{\partial q_{k}}{\partial x_{k}}\right)+D_{c} S_{k} u_{k}, \\
& S_{i}=Q_{c} E_{i},
\end{aligned}
$$

where $x_{i}$ is the position vector, $u_{i}$ is the velocity vector, $q_{i}$ is the heat flux vector, $\rho$ is the density, $p$ is the static pressure, $e$ is the total energy per unit volume, $\tau_{i j}$ is the stress tensor, $\delta_{i j}$ is the Kronecker delta, $S_{i}$ is the body force vector, $Q_{c}$ is the electric charge, $E_{i}$ is the electric field vector, and $t$ denotes time; note that these are all nondimensional forms. Here, $x=x_{1}, y=x_{2}, u=u_{1}$, and $v=u_{2}$. The nondimensional parameters, namely, the Reynolds number (Re), the Prandtl number $\left(P_{r}\right)$, and the Mach $(M)$ number, are defined as follows:

$$
R e=\frac{\rho_{r e f} U_{r e f} L_{r e f}}{\mu_{r e f}}, P_{r}=\frac{\mu_{r e f} C_{p}}{\kappa_{r e f}}, M=\frac{U_{r e f}}{a_{r e f}},
$$

where $\mu_{r e f}$ is the viscosity, $L_{r e f}$ is the length, $U_{r e f}$ is the velocity, $a_{r e f}$ is the speed of sound, $C_{p}$ is the specific heat at constant pressure, and $\kappa_{r e f}$ is the thermal conductivity; the subscript ref indicates the reference values. In Eqs. 2 and 3 ., the final terms of the right-hand side represent the momentum and energy added to a unit volume by the body force model. The body force model will be explained in the next subsection. This system is closed, and so the ideal gas equation of state is written as follows:

$$
p=(\gamma-1)\left(e-\frac{1}{2} \rho u_{k} u_{k}\right)
$$

where $\gamma$ is the ratio of specific heats.

\subsubsection{Body force model of a plasma actuator}

As mentioned in the previous subsection, the body forces provided by the plasma actuator are modeled by adding source terms, namely $D_{c} S_{i}$ and $D_{c} u_{k} S_{k}$, to the NS equations. In this study, we used the Suzen-Huang body force model (Suzen, et al., 2005) to obtain the spatial distribution of $S_{i}$. In this model, the nondimensional plasma actuator body force vectors $\left(S_{i}\right)$ are computed by multiplying the electric charge $\left(Q_{c}\right)$ by the electric field vector $\left(E_{i}\right)$, since the Maxwell equations were solved to determine the electric field due to the applied AC voltage at the electrodes, and an additional equation was solved to determine the charge density for the plasma. Further details can found in the reference (Suzen, et al., 2005). A nondimensional parameter $\left(D_{c}\right)$ is defined as follows:

$$
D_{c}=\frac{Q_{c, r e f} E_{r e f} L_{r e f}}{\rho_{r e f} U_{r e f}^{2}},
$$

where the subscript ref indicates the reference values. $Q_{c, r e f}$ and $E_{\text {ref }}$ were set to the maximum values of $Q_{c}$ and $E$, respectively, as computed by Suzen, et al., (2005). Note that the previous study (Gaitonde, et al., 2005) described it as the ratio of the electrical force of the fluid to its inertial force. The contours of the spatial distribution of the body force and 
the body force vectors are shown in Fig. 3. Note that the component of body force in the spanwise direction is assumed to be zero. To obtain the temporal variation in the body force distribution, we multiply the body forces $S_{i}$ by $\sin ^{2}\left(2 \pi F_{b a s e} t\right)$. This model is based on the assumption that the temporal variation of the body force can be characterized as push/push (Font, et al., 2010).

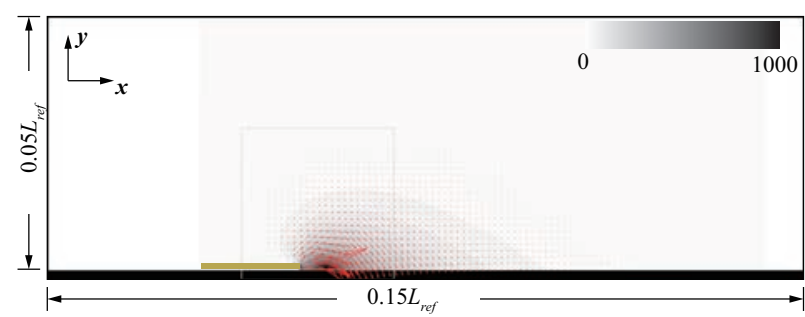

(a) overview

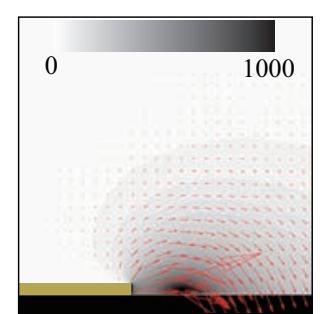

(b) close-up view near the exposed electrode

Fig. 3 Spatial distribution of the plasma actuator body force model (Suzen, et al., 2005).

\subsubsection{Spatial and temporal discretization}

The spatial derivatives of the convective and viscous terms and the symmetric conservative metrics (Vinokur and Yee, 2002, Deng, et al., 2013, Abe, et al., 2014) and Jacobian (Vinokur and Yee, 2002, Deng, et al., 2013, Abe, et al., 2014) were evaluated by a sixth-order compact difference scheme (Lele, 1992). Near the boundary, second-order explicit difference schemes were utilized. Tenth-order filtering (Lele, 1992, Gaitonde and Visbal, 2000) was used with a filtering coefficient of 0.495 . For time integration, lower-upper symmetric alternating direction implicit and symmetric Gauss-Seidel methods (Fujii 1998, Nishida and Nonomura, 2009) were adopted. To ensure time accuracy, a backward second-order difference formula and five inner iterations (Charkravarthy, 1984) were used. The computational time step was set to $4.0 \times 10^{-5}$ in nondimensional time. The maximum local Courant number was approximately 2.0, and zero gradient conditions were adopted at the outer boundaries. On the surface of the flat plate, no-slip and adiabatic conditions were imposed. At the inlet, zero velocities are assigned.

\subsubsection{Computational grids}

Figure 4 illustrates a computational grid. We used the sufficiently large computational domain so as to make sure the outer boundary did not affect the computed flow structures. To clarify the setup, in Fig. 4, the solid orange rectangle indicates the exposed electrode of the plasma actuator (note that it does not physically exist in the computational domain); its width is $0.02 L_{r e f}$, and its height is $0.001 L_{r e f}$. Near the exposed electrode, the grid is distributed uniformly. To examine the effect of the resolution of the grid on the structure of the flow, we considered three two-dimensional grids, as follows: coarse $(513 \times 260)$, intermediate case $(873 \times 416)$, and fine case $(1583 \times 722)$. The velocity profiles matched well when we used the intermediate or fine grids, and we used the intermediate grid for the following simulations. To capture the flow details in the plasma area, we used a finer grid in the plasma area and near the wall. The finer spacing was $0.0002 L_{r e f}$ $\left(2 \times 10^{-5} \mathrm{~m}\right)$. We used five grid points in the direction normal to the wall to discretize the height of the exposed electrode $\left(1 \times 10^{-4} \mathrm{~m}\right)$ and to resolve the flow induced by the plasma actuator, near the exposed electrode.
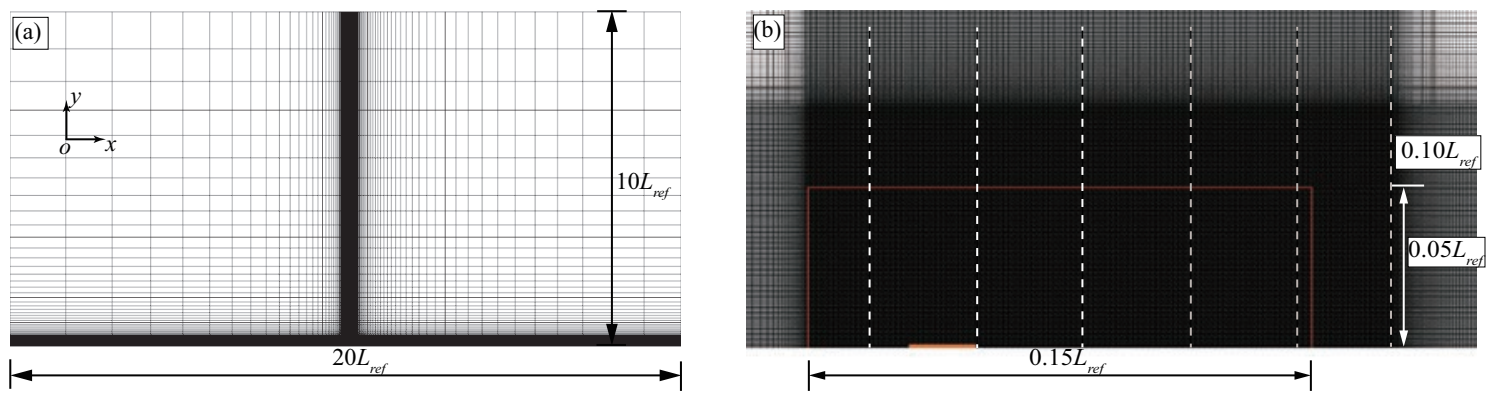

Fig. 4 Schematic of the computational domain. (a) Overview, and (b) near the plasma actuator. 


\subsection{Experimental setup}

The plasma actuator consists of two dielectric electrodes (see Fig. 5), each $50 \mu \mathrm{m}$ thick (3M, Cu-35C), and two polyimide dielectric layers, each $80 \mu \mathrm{m}$ thick (Teraoka Seisakusho, 650S; $50 \mu \mathrm{m}$ thick). We note that this plasma actuator uses two layers, unlike the one-layer configuration discussed above. The gap between the two electrodes was $0.08 \mathrm{~mm}$. The upper exposed electrode was $2.0 \mathrm{~mm}$ wide, and the insulated electrode was $6.0 \mathrm{~mm}$ wide. The overlapping design (Roth and Dai, 2006) was used and the gap length was $1.0 \mathrm{~mm}$. A $150 \mathrm{~mm}$ long actuator was installed on a glass-fiber reinforced plastic board, and this was placed inside the chamber, as shown in Fig. 6. The geometrical configuration of the plasma actuator was not identical to that used for the computations, but we believe that this small difference did not significantly impact the results. It should be noted that based on the results of Teflon and PMMA actuators reported in the previous studies (Roth and Dai, 2006, Forte, et al., 2007), it was found that the range of the gap considered in this study (from $-1 \mathrm{~mm}$ to $1 \mathrm{~mm}$ ) has small influence on the maximum velocity of induced flows under the operating conditions considered here.

(a) Computation

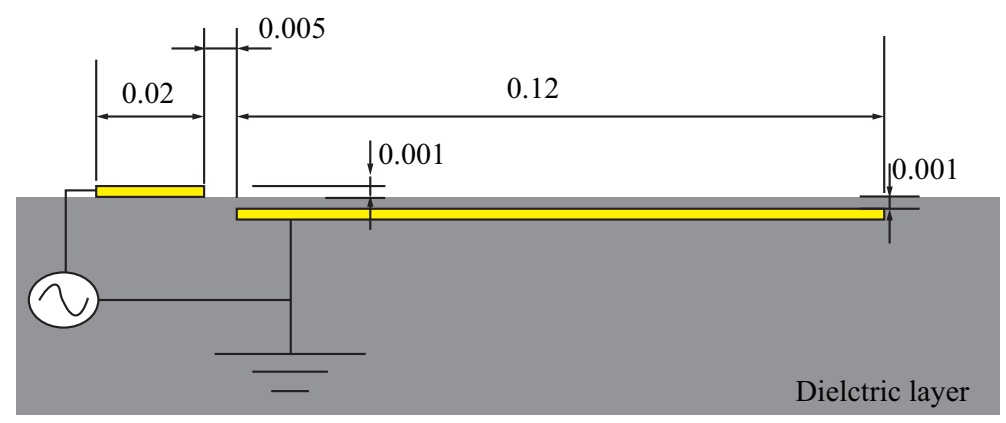

(b) Experiment

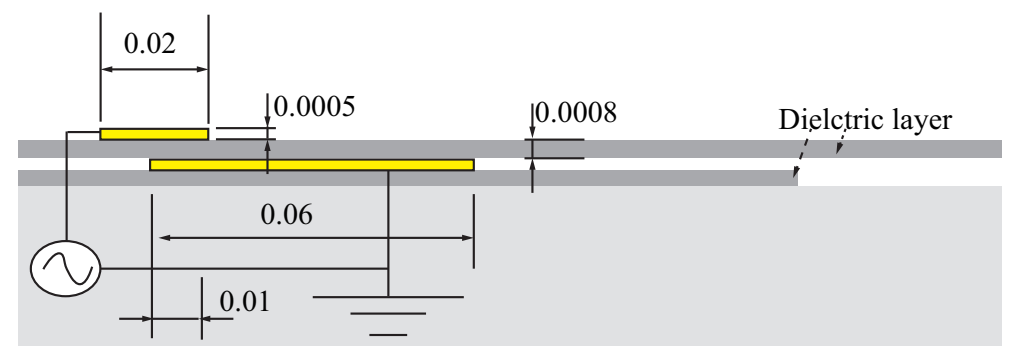

Fig. 5 Configuration of the plasma actuator (a) used for the computations, and (b) used in the experiment.

\subsubsection{Schlieren imaging}

Schlieren imaging was used to visualize density variations in the air; such images were taken by a high-speed camera (Phantom v311 with a Sigma APO300-800 mm F5.6 lens). The recording frequency was 10,000 Hz. Since a horizontal knife edge is inserted from above, low brightness indicates compression and high intensity indicates expansion in the upward direction. Note that the brightness is proportional to the density gradient in the vertical direction. Further details of schlieren imaging can be found in the references (Sekimoto, et al., 2014, Sekimoto, 2015).

\subsubsection{Particle image velocimetry}

Air velocities were measured using PIV. A high-volume glycerin liquid droplet (10 $\mu \mathrm{m}$ diameter) generator was used for seeding, and the seed particles were illuminated by a $2 \mathrm{~W}$ continuous-wave laser (RayPower 2000). The distance from the laser to the surface was $60 \mathrm{~mm}$. Images of the particle were collected by a high-speed (2500 fps) camera with an intensifier. After obtaining a series of pictures, post-processing software (DynamicStudio by Dantec Dynamics) was used to calculate the velocity field. PIV images were acquired in pairs at $2500 \mathrm{~Hz}$, and the time between the two images in each pair was $800 \mu \mathrm{s}$. The adaptive correlation method was used to derive the instantaneous velocity field from each pair of images. The final interrogation area was $8 \times 8$, with an overlap ratio of $50 \%$; the number of refinement steps was 3 . This corresponds to a resolution of $0.496 \mathrm{~mm} \times 0.496 \mathrm{~mm}$. To reduce the noise, 450 instantaneous velocity fields were averaged. Interested readers can find additional detailed information in the references (Sekimoto, et al., 2012, Sekimoto, 


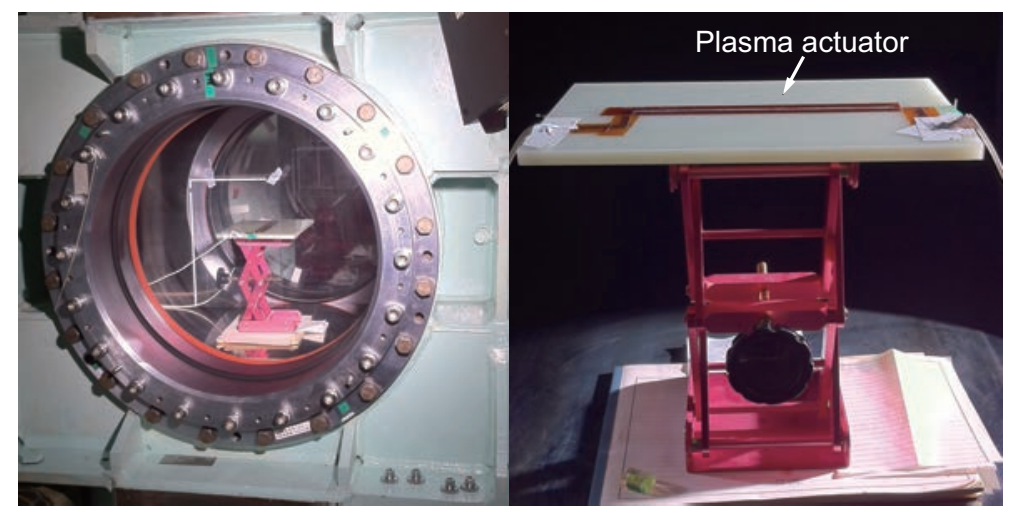

Fig. 6 Experimental facilities for schlieren imaging.

2015).

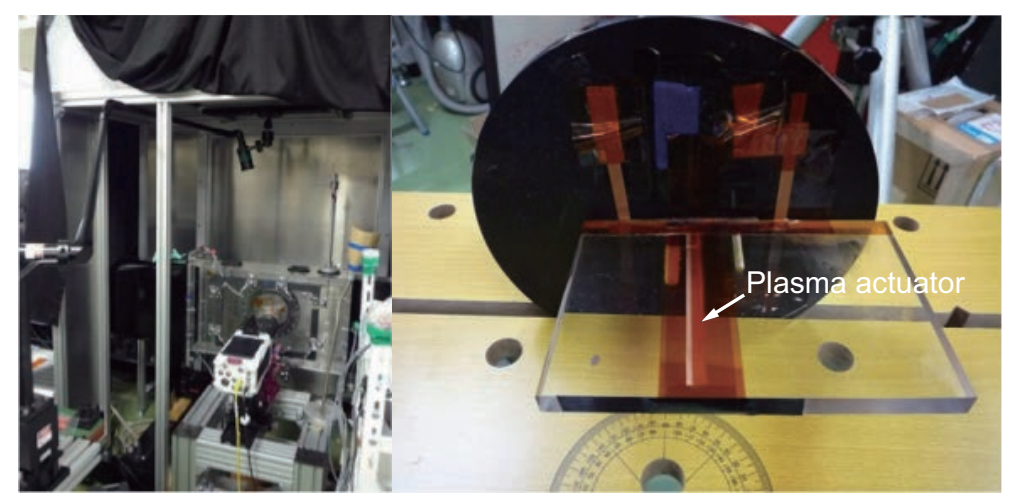

Fig. 7 Experimental facilities for PIV.

\subsection{Averaging and time period for comparison}

The time period for averaging the results was from $0.02 \mathrm{~s}\left(2 T^{+}\right)$after the discharge until $0.2 \mathrm{~s}\left(20 T^{+}\right)$afterwards. These were compared with the instantaneous flow fields from $0.18 \mathrm{~s}\left(19 T^{+}\right)$to $0.20 \mathrm{~s}\left(20 T^{+}\right)$. Note that $T^{+}$is $1 / F^{+}$, and $F^{+}$was selected to be $1(100 \mathrm{~Hz})$. We carried out a priori tests with PIV to assess the effect of the averaging period on the maximum velocity of the wall jet; we considered periods of $0.18,0.28$, and $0.38 \mathrm{~s}$, and we used a $V_{p p}$ of $7 \mathrm{kV}$. The results showed that the maximum wall jet velocities were within $20 \%$ of the average value.

\section{Results and Discussion}

The computed time-averaged induced flow fields were compared with the density variation and the profile of the wall jet, in order to investigate the impact of $D_{c}$ on the predicted flow structures when $F^{+}$was $1(100 \mathrm{~Hz})$. Next, the effects of $F^{+}$on the time-averaged induced flow structure was analyzed. Finally, the impact of $F^{+}$on the instantaneous induced flow structure was discussed. The current study focuses on the flow induced by the plasma actuator operated with burst modulations, but in Appendix, we present a comparison of induced flow structures under continuous actuation.

\subsection{Time-averaged flow fields}

\subsubsection{Burst frequency of $100 \mathrm{~Hz}\left(F^{+}=1\right)$}

For $F^{+}=1(100 \mathrm{~Hz})$, Fig. 8 compares the experimental schlieren photography results with the computed ones. The five computed results are each associated with different values of $D_{c}$, while all of the experimental images correspond to $F^{+}=1(100 \mathrm{~Hz}), \mathrm{BR}=10 \%$, and $V_{p p}=7 \mathrm{kV}$. The change in $D_{c}$ results in different flow structures being induced by the plasma actuator. Numerical simulations with the current simple body force model are in qualitative agreement with the experimental results in the $D_{c}$ range considered here.

The maximum velocity of the induced wall jet $\left(u_{i, \max }\right)$ as a function of $D_{c}$ is shown in Fig. 9; the values measured by PIV are also included. The two lines in Fig. 9 each correspond to a different wall-parallel position. One is measured at the 


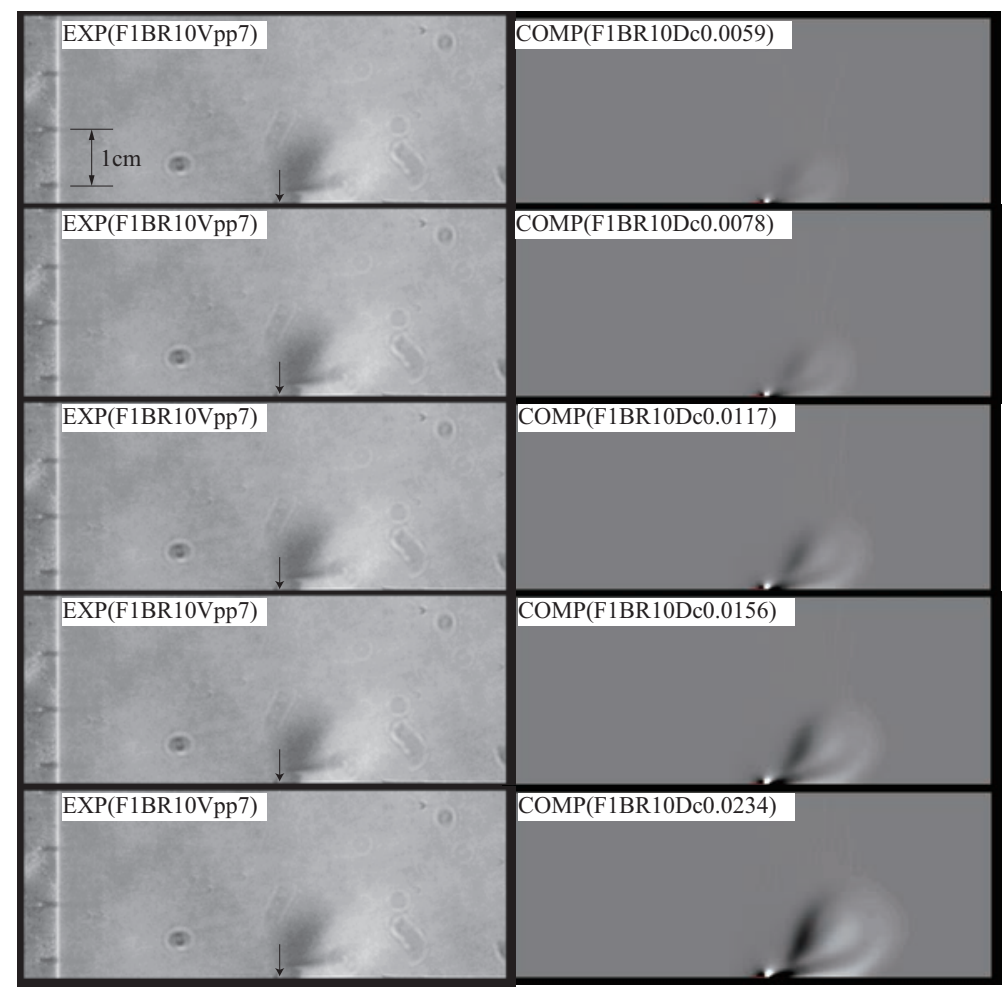

Fig. 8 Time-averaged flow structures induced by the plasma actuator associated with $F^{+}$of $1(100 \mathrm{~Hz})$. Left, experimental schlieren image; Right, computational schlieren image.

location of the maximum wall jet near the exposed electrode, and the other is at $x / L_{r e f}=0.05$, which is the same position as in the experiment. The maximum induced wall jet velocity increases with increasing $D_{c}$ at all locations. The wallnormal distances at these same wall-parallel positions are shown as a function of $D_{c}$ in Fig. 10; the values measured by PIV are also included. As $D_{c}$ increases, the maximum wall-normal distance for the wall jet decreases. These observations indicate that as $D_{c}$ increases, the generated wall jet becomes stronger and thinner. Moreover, the maximum wall-normal distances are more than five times the height of the plasma actuator.

Figure 11 shows a comparison of the profiles for the velocity of the wall-parallel flow at various wall-parallel positions. Most of profiles are in agreement with the PIV data, except near the right edge of the exposed electrode. The velocity there $\left(x / L_{r e f}=0.0\right)$ is overpredicted. This may be due to the original distribution of the body force, or it may be due to measurement error in the experiment; we hope to determine this in the near future. However, looking at $x / L_{r e f}=0.05$ in Figures 9, 10, and 11, it is clear that with $D_{c}=0.0117$, the profile, the maximum velocity of the wall-parallel flow, and the predicted position are in reasonable agreement with the experimental results. Thus, when $D_{c}=0.0117$, the density variation of the overall flow structures is in agreement with the experimental results, as shown in Fig. 8.

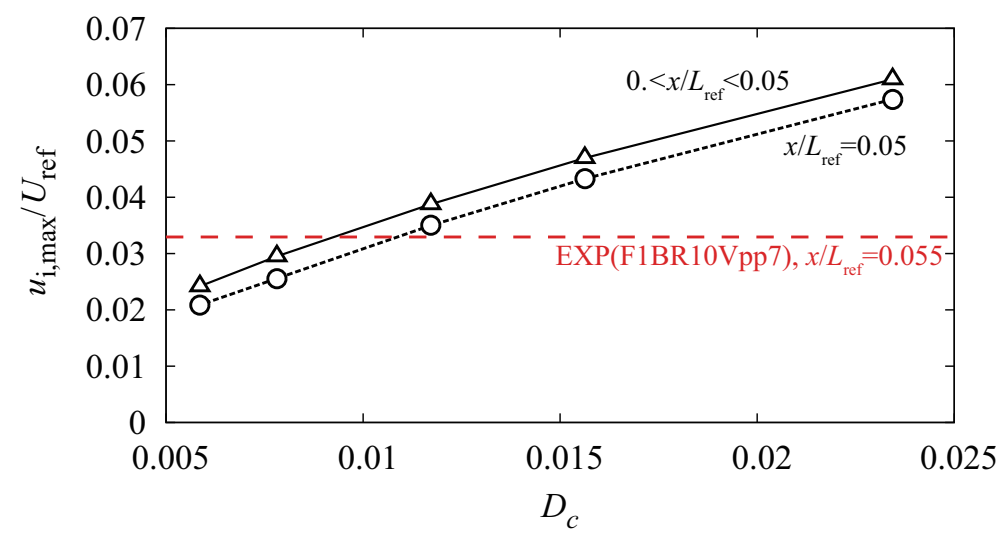

Fig. 9 The maximum velocity of the wall-parallel flow for the body force model as a function of $D_{c}$ associated with $F^{+}=1(100 \mathrm{~Hz})$. Lines with open symbols are the computed results, and the red dashed line is the experimental data. 


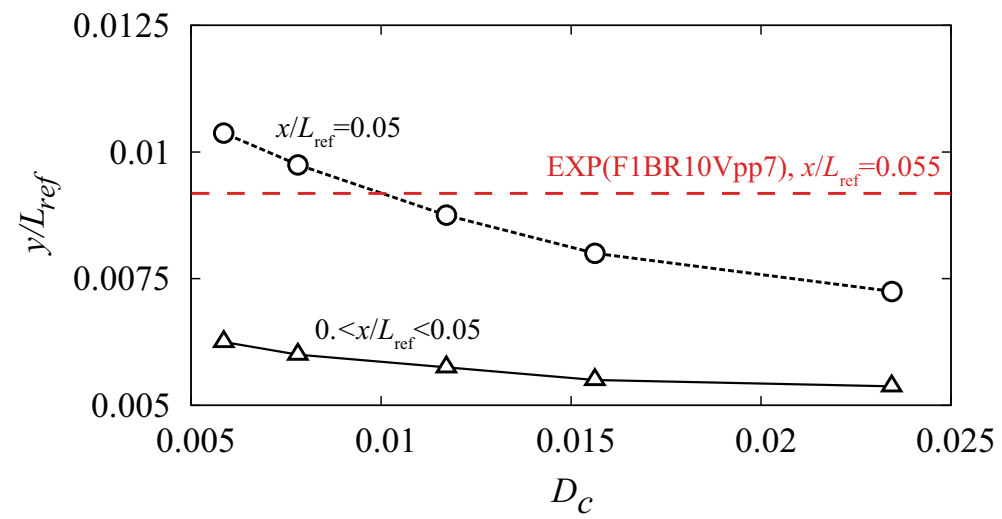

Fig. 10 The wall-normal distance at the position where the maximum velocity of the wall-parallel flow induced by the body force model appears, as a function of $D_{c}$ associated with $F^{+}=1(100 \mathrm{~Hz})$. Lines with open symbols are the computed results, and the red dashed line is the experimental data.

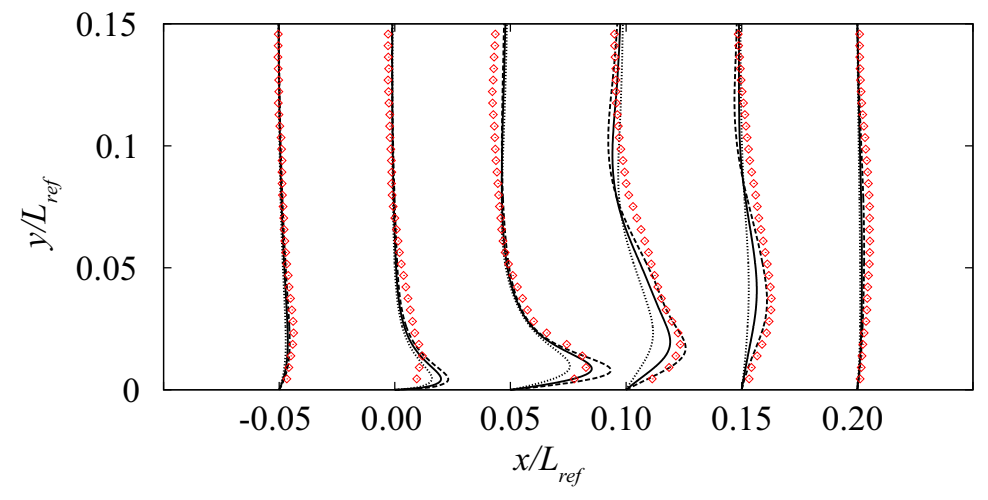

Fig. 11 Profiles of the velocity of the wall-parallel flow for the body force model associated with $F^{+}=1(100$ $\mathrm{Hz}$ ). Dotted, solid, and dashed lines indicate the computed results with $D_{c}=0.078,0.0117$, and 0.0156, respectively. Red diamonds correspond to the PIV data with $V_{p p}=7 \mathrm{kV}$.

\subsubsection{Burst frequency of $500 \mathrm{~Hz}\left(F^{+}=5\right)$}

Comparisons of the cases of $F^{+}=5(500 \mathrm{~Hz})$ are presented in Figs. 12 and 13. As in the case with $F^{+}=1$, it is found that the flow fields are in good agreement with the experimental results.

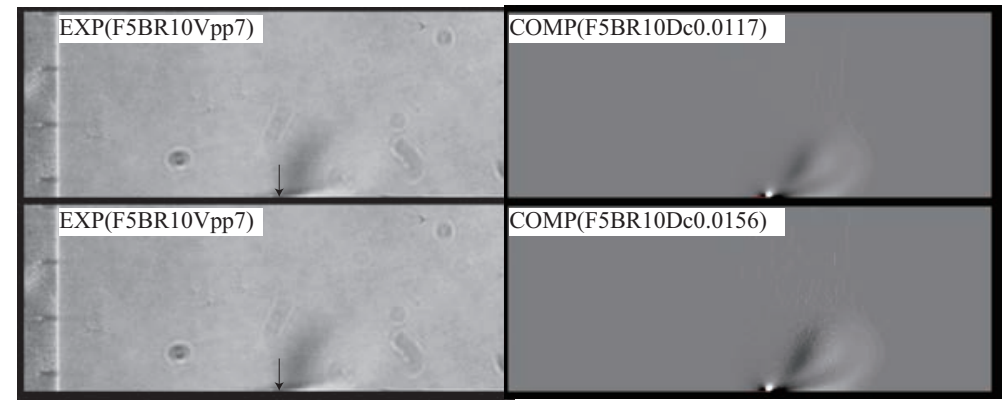

Fig. 12 Time-averaged flow structures induced by the plasma actuator associated with $F^{+}=5(500 \mathrm{~Hz})$. Left, experimental schlieren image; right, computational schlieren image.

\subsubsection{Burst frequency of $1,000 \mathrm{~Hz}\left(F^{+}=10\right)$}

Comparisons for various cases with $F^{+}=10(1,000 \mathrm{~Hz})$ are presented in Figs. 14 and 15. As in the case with $F^{+}$of 1 and 5 , it is seen that the flow fields are in good agreement with the experimental results.

\subsubsection{Effects of burst frequency}

Profiles of the velocity of the induced flow and the fluctuation in the wall-parallel direction are shown in Fig. 16. It 


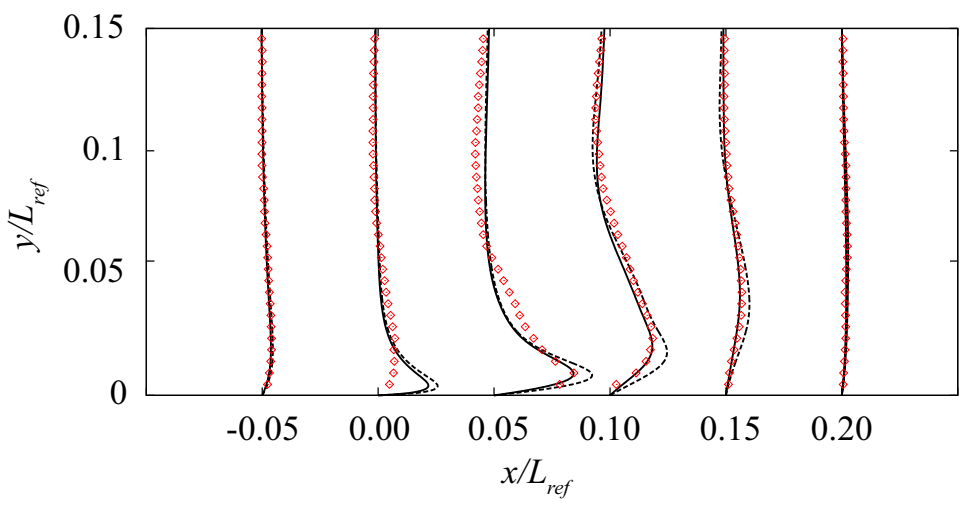

Fig. 13 Profiles of the time-averaged velocity of the wall-parallel flow induced by the body force model associated with $F^{+}=5(500 \mathrm{~Hz})$. Solid and dashed lines indicate the computed result, with $D_{c}=$ 0.0117 , and 0.0156 , respectively. Red diamonds correspond to the PIV data with $V_{p p}=7 \mathrm{kV}$.

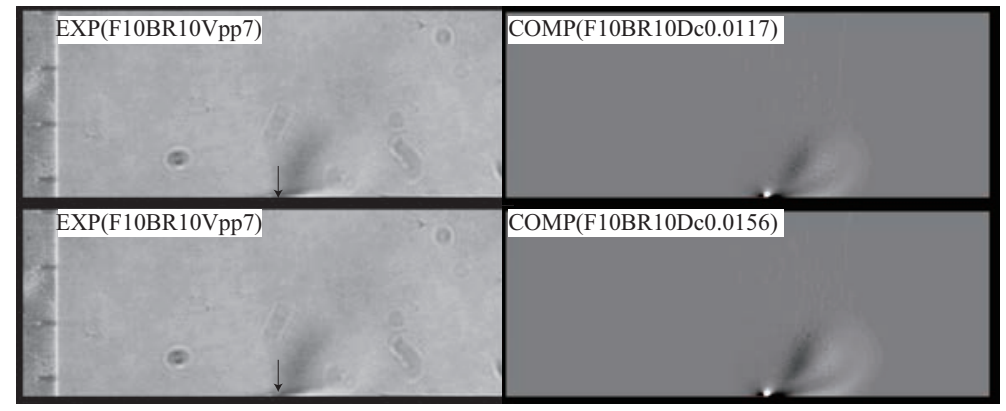

Fig. 14 Time-averaged flow structures induced by the plasma actuator associated with $F^{+}=10(1,000 \mathrm{~Hz})$. Left, experimental schlieren image; Right, computational schlieren image.

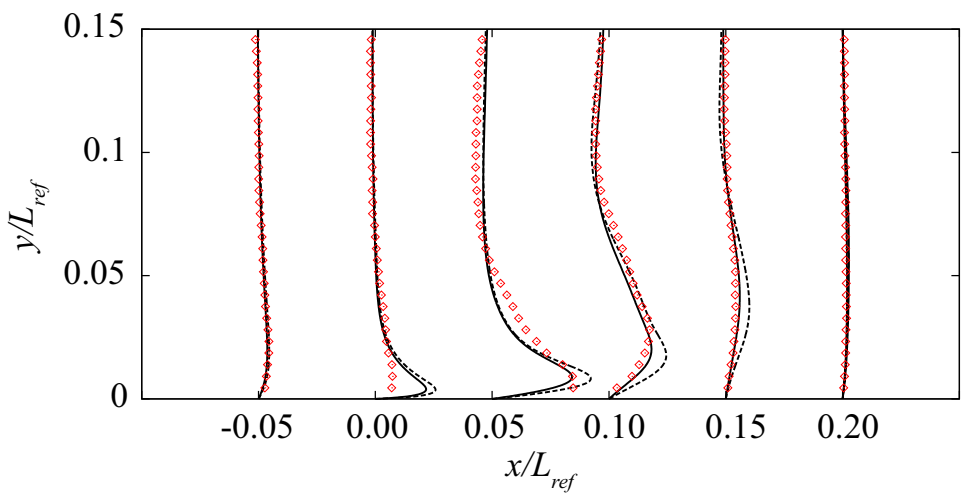

Fig. 15 Profiles of time-averaged velocities of the wall-parallel jets induced by the body force model associated with $F^{+}=10(1,000 \mathrm{~Hz})$. Solid and dashed line indicate the computed results, with $D_{c}=0.0117$ and 0.0156 , respectively. Red diamonds correspond to the PIV data with $V_{p p}=7 \mathrm{kV}$.

can be seen that in the frequency range considered here, the velocity of the induced wall-parallel flow is not dependent on $F^{+}$. The primary difference is the amplitude of the induced flow oscillations near the exposed electrode. Similar observations have been reported by Benard and Moreau (2010).

\subsection{Instantaneous flow fields}

Figure 17 shows the profiles of the instantaneous velocity of the wall-parallel flow at selected times for $F^{+}$of 1,5 , and 10. It can be seen that the body force produces flow near the exposed electrode (i.e., $x / L_{\text {ref }}=0.0 \sim 0.05$ ) when the plasma actuator is switched on, and the flow varies in time. Moreover, looking at the velocity of the wall-parallel flow over time at specific positions, it can be seen that the time variation of the wall-parallel velocity decreases as the burst frequency increases (see Fig. 18). This suggests that the duration during which the plasma actuator is switched off plays an important role in determining the amplitude of fluctuations in the velocity. This is consistent with the findings of previous experimental studies (Benard and Moreau, 2009, 2014). 

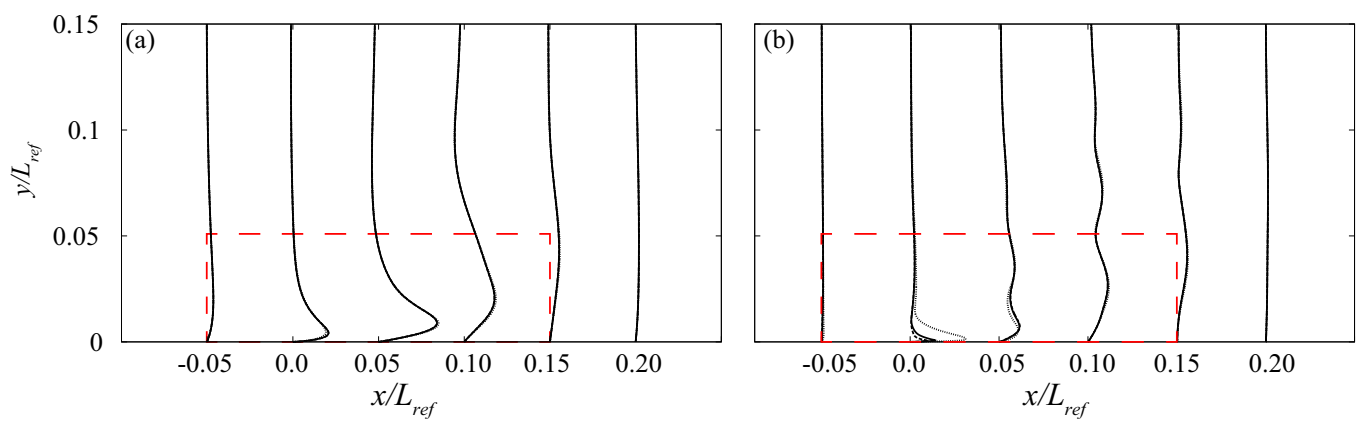

Fig. 16 Comparison of (a) the velocity of the wall-parallel flow, and (b) the distribution of the fluctuations, as induced by the plasma actuator body force. Dotted, solid, and dashed lines denote $F^{+}=1(100 \mathrm{~Hz}), 5(500$ $\mathrm{Hz})$, and $10(1,000 \mathrm{~Hz})$, respectively. The body forces were applied to the region inside the rectangles indicated by the red dashed lines.
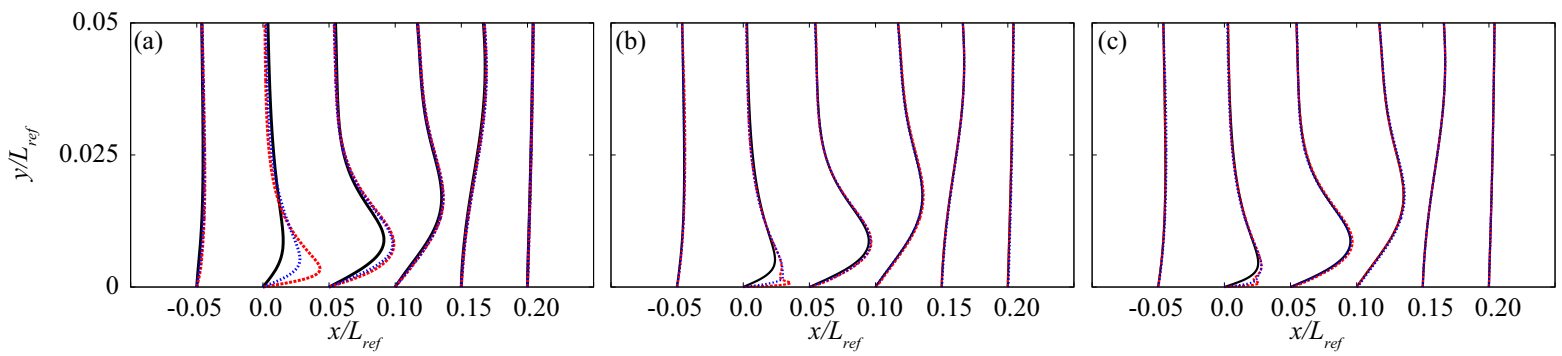

Fig. 17 Profiles of the instantaneous velocity of the wall-parallel flow at selected positions and times. (a) $F^{+}=$ $1(100 \mathrm{~Hz})$; (b) $F^{+}=5(500 \mathrm{~Hz}) ;(\mathrm{c}) F^{+}=10(1,000 \mathrm{~Hz})$. Solid black, red dashed, and blue dotted lines correspond to $T_{1}=1 / F^{+}, T_{2}=\mathrm{BR} / F^{+}$, and $T_{3}=2 T_{2}$, respectively.
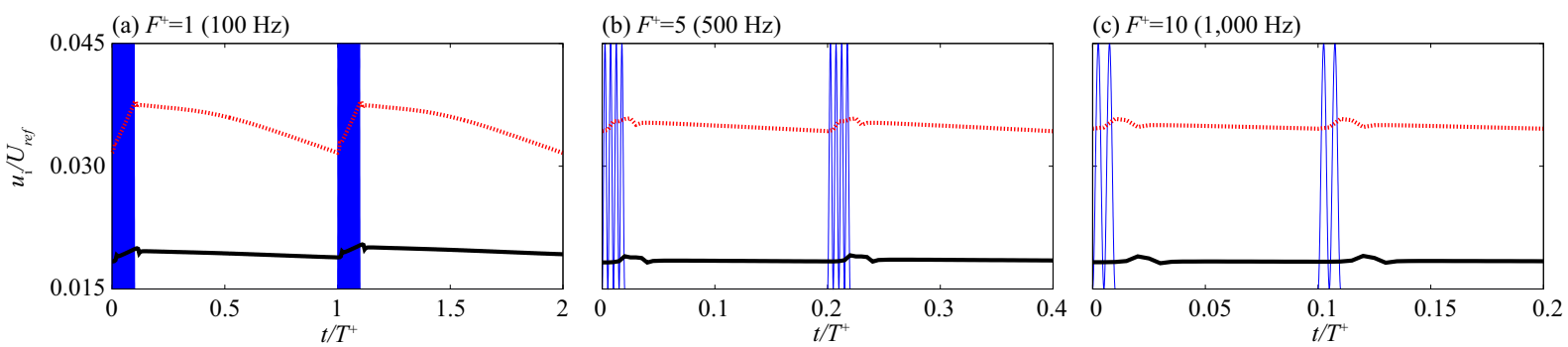

Fig. 18 Profiles of the velocity of the wall-parallel flow over time at selected wall-parallel positions. (a) $F^{+}=1$ $(100 \mathrm{~Hz}) ;\left(\right.$ b) $F^{+}=5(500 \mathrm{~Hz}) ;\left(\right.$ c) $F^{+}=10(1,000 \mathrm{~Hz})$. Red dashed and black solid lines correspond to the results at $\left(x / L_{r e f}=0.05, y / L_{r e f}=0.00875\right)$ and $\left(x / L_{r e f}=0.1, y / L_{r e f}=0.00875\right)$, respectively. Blue lines indicate where the bursting waves are input.

PSDs of the velocity of the wall-parallel flow at the selected locations are shown in Fig. 19. The horizontal axis indicates the Strouhal number, which is defined as $S t=f_{u} L_{r e f} / U_{r e f}$, where $f_{u}$ is the fluctuating frequency of the wallparallel flow. The number of points taken for the fast Fourier transform was $2^{11}$, and the PSD presented here is for the unfiltered signals. The velocity signal has strong components not only at the main frequency of the bursts but also for several of its harmonics. At the location of the maximum velocity of the time-averaged wall-parallel flow, the average power of the signal was computed by using the rectangle rule to approximate the integral of the PSD. We found that 91.7\%, 94.8\%, and $94.9 \%$ of the average signal power was contained in the first three harmonics when $F^{+}=1,5$, and 10 , respectively. At $x / L_{r e f}=0.1$, the high-frequency components $(S t>10)$ still exist, while the PSDs corresponding to the burst frequency are significantly damped out. It should be mentioned here that these multiple frequencies can contribute to flow control mechanisms.

\section{Summary}

The flow induced by a plasma actuator operated with burst modulations in quiescent air was computed by solving the Navier-Stokes equations with varying body force terms. The time-varying body-force distribution adopted in this study is based on the Suzen-Huang body force model (Suzen, et al., 2005) and simple time variation. We validated 


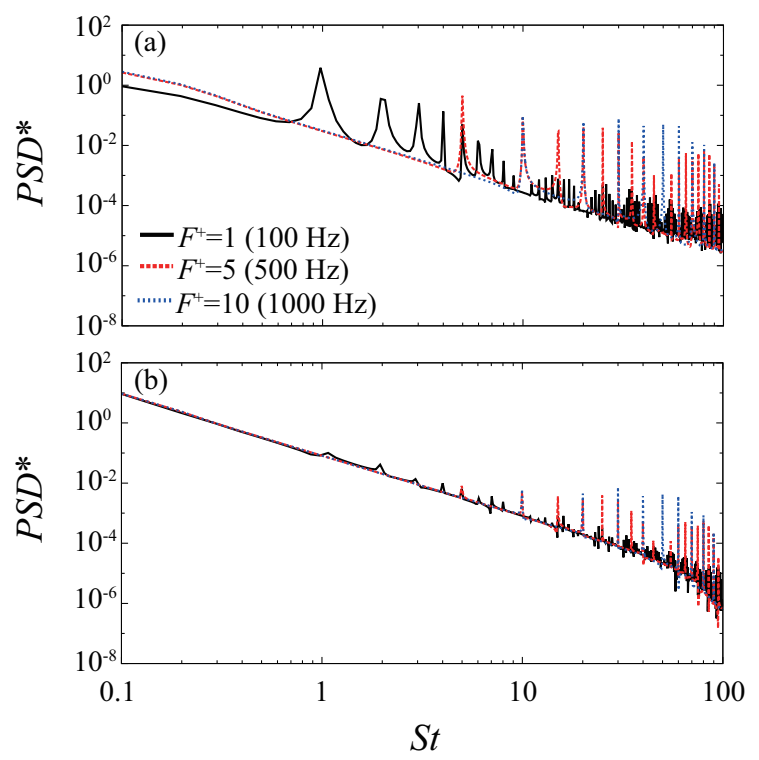

Fig. 19 Power spectral densities of the velocity of the wall-parallel flow for $F^{+}=1(100 \mathrm{~Hz}), 5(500 \mathrm{~Hz})$, and $10(1,000 \mathrm{~Hz})$ at selected positions: (a) $\left(x / L_{r e f}=0.05, y / L_{r e f}=0.00875\right)$ and (b) $\left(x / L_{r e f}=0.1\right.$, $\left.y / L_{r e f}=0.00875\right)$.

our body force model through a careful comparison of the computed results with the experimental results. We found that when we used an appropriate value of the nondimensional parameter $\left(D_{c}\right)$, then the simple time-varying body force model is able to qualitatively predict the induced flow structures and the wall jet associated with burst modulations. With an appropriate $D_{c}$, we obtained a quantitative match between the simulated and experimentally measured structures of the maximum velocity of the wall-parallel flow. We also investigated the effects of the burst frequency on the induced flow structures. We computed the development of the wall jet induced by the plasma actuator, and the magnitude of the maximum time-averaged wall jet was the same for all cases that we considered. Most of results are consistent with the observations reported for previous experiments (Benard and Moreau, 2010, Kotsonis and Veldhuis, 2010), and this lends further support to our computed results. As an area of future work, we intend to investigate the characteristics of the flow induced by a plasma actuator with burst modulations in the free-stream.

\section{Acknowledgement}

Part of this research was conducted on the K computer and other computers of the high performance computing infrastructure (HPCI) system, and this support was provided by RIKEN the advanced institute for computational science and the information technology center at the University of Tokyo through the HPCI system research project (Project ID: hp120296, hp130001, hp140207). The authors would like to thank Dr. Hiroyuki Nishida for fruitful discussions.

\section{Appendix: Flow structures induced by continuous actuation}

Comparisons of the time-averaged flow fields induced by the plasma actuator with continuous actuation are shown in Fig. 20. Both the nondimensional $\left(D_{c}\right)$ and the applied voltage $\left(V_{p p}\right)$ are varied. It can be seen that an increase in $V_{p p}$ enlarges the area of the wall jet and the size of the initial vortex. This trend can also be seen in the computed results. However, the flow structure looks different when $D_{c}$ increases to more than 0.5. Qualitatively, the computed result when $D_{c}=0.0039$ agrees with the experimental data when $V_{p p}=9 \mathrm{kV}$, and the computed results when $D_{c}=0.0078$ and 0.0156 are in agreement with the experimental data when $V_{p p}=10 \mathrm{kV}$. The relationship between the maximum time-averaged velocity of the wall jet and $D_{c}$ is shown in Fig. 21; the wall-normal and wall-parallel distances at the position where this velocity is greatest depends on $D_{c}$, and this is also shown in Fig. 21. It can be seen that the maximum time-averaged velocity of the wall jet increases with increasing $D_{c}$, and the position at which this maximum velocity occurs moves towards the wall and away from the exposed electrode. This is also similar to what was observed in the experiments. In short, a simple time-varying body force is able to qualitatively reproduce the induced flow structures and the wall jet associated with continuous actuation. 


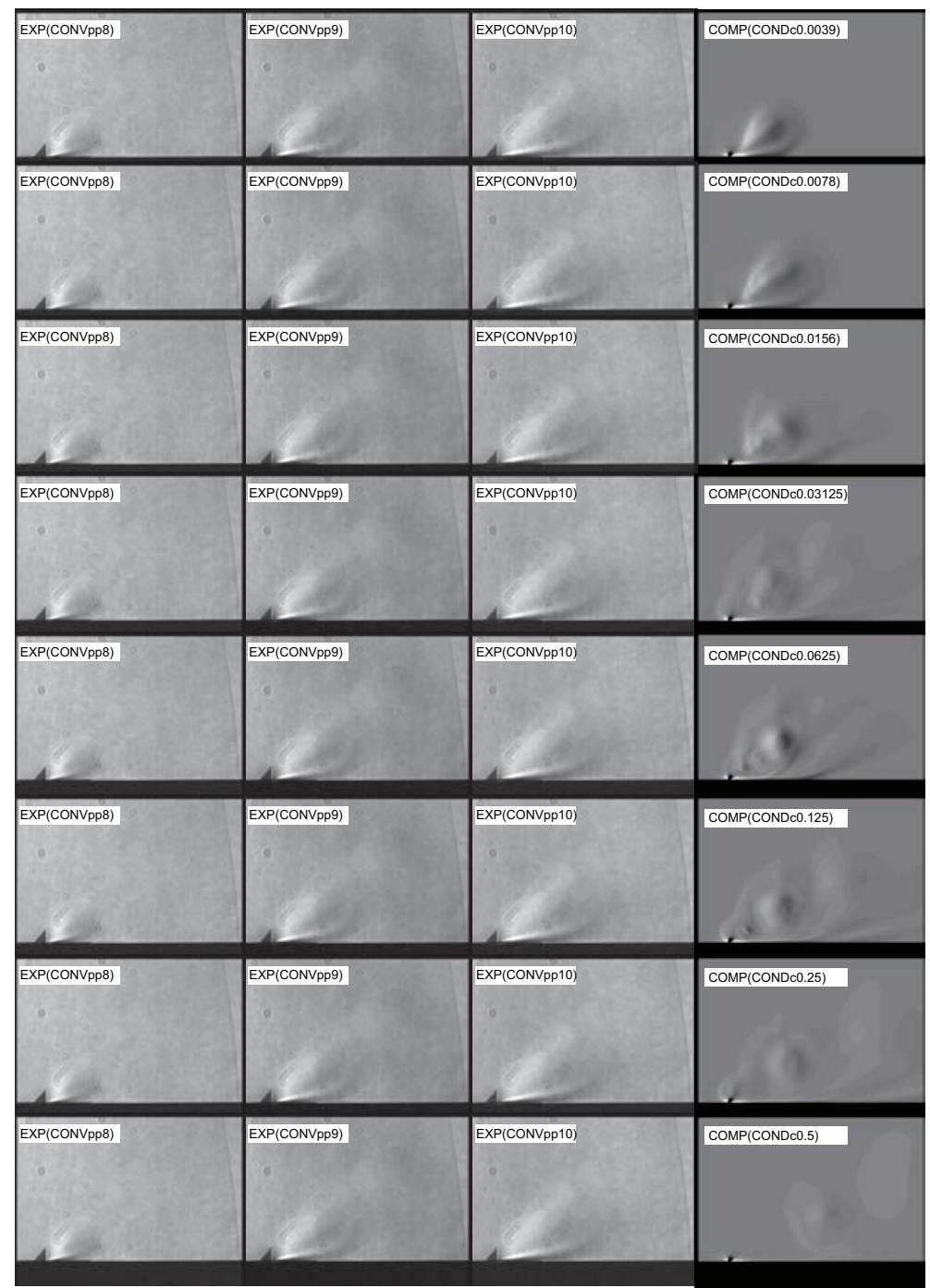

Fig. 20 Time-averaged flow structures induced by the plasma actuator with continuous excitation. Three left columns, experimental schlieren image; Right column, computational schlieren image.
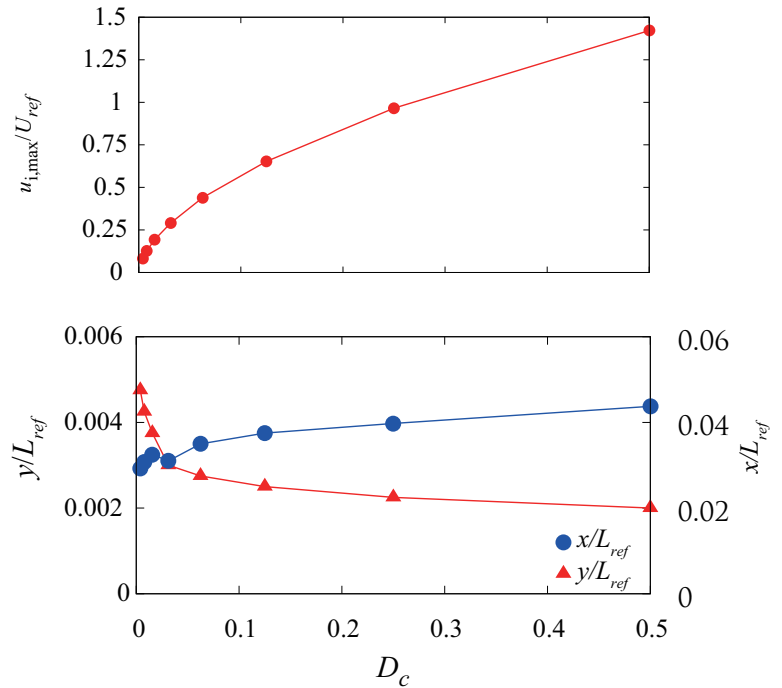

Fig. 21 The maximum time-averaged velocity of the wall jet induced by the plasma actuator body force as a function of the strength of the body force model $\left(D_{c}\right)$ and associated with continuous actuation. The wall-normal and wall-parallel distances at the position at which the velocity is greatest are indicated. 
Since the body force is distributed over a limited region, how the energy is transferred to the induced wall jet is of interest. We can obtain a simple estimate of the work done by the body force $\left(W_{b f}\right)$ by multiplying the body force by the time-averaged induced flow, and a simple estimate of the work done by the total pressure $\left(W_{t p}\right)$ by multiplying the total pressure by the time-averaged induced flow:

$$
\begin{aligned}
& W_{b f}=D_{c} \int_{x_{\min }}^{x} \int_{y_{\min }}^{y_{\max }}\left(S_{x} u+S_{y} v\right) d y d x \\
& W_{t p}=\int_{y_{\min }}^{y_{\max }} p_{t} u d y
\end{aligned}
$$

where the total pressure $p_{t}=p+\frac{1}{2} \rho\left(u_{k} u_{k}\right)$. In addition, when the flow velocity and its fluctuation are small, if we assume the flow is incompressible, the viscous dissipation function $(\Psi)$ can be calculated:

$$
\Psi=\int_{x_{\min }}^{x} \int_{y_{\min }}^{y_{\max }}\left(2 \mu\left(e_{i j}-\frac{1}{3} e_{k k} \delta_{i j}\right)^{2}\right) d y d x
$$

where $e_{i j}$ is the shear velocity tensor $\frac{1}{2}\left(\partial u_{i} / \partial x_{j}+\partial u_{j} / \partial x_{i}\right)$. Figure 22 shows these three terms as functions of the wallparallel direction. At first, the average energy transfer at the streamwise position $\left(x / L_{r e f}\right)$ is computed using the rectangle approximation of the integral for each term in the wall-normal direction, and then $W_{b f}$ and $W_{t p}$ are summed from upstream to downstream. It can be seen that $W_{b f}$ is at a maximum near $x / L_{r e f}=0.075$. $W_{t p}$ quickly increases from the location of the exposed electrode, and it reaches a maximum when $x / L=0.03$. We also see that $\Psi$ gradually increases, and it dominates when $x / L>0.07$. At the point where the time-averaged wall jet is at a maximum, $W_{t p}$ is $66 \%$ of the $W_{b f}$, and the viscous dissipation function is approximately $30 \%$ of the work done by the body force.

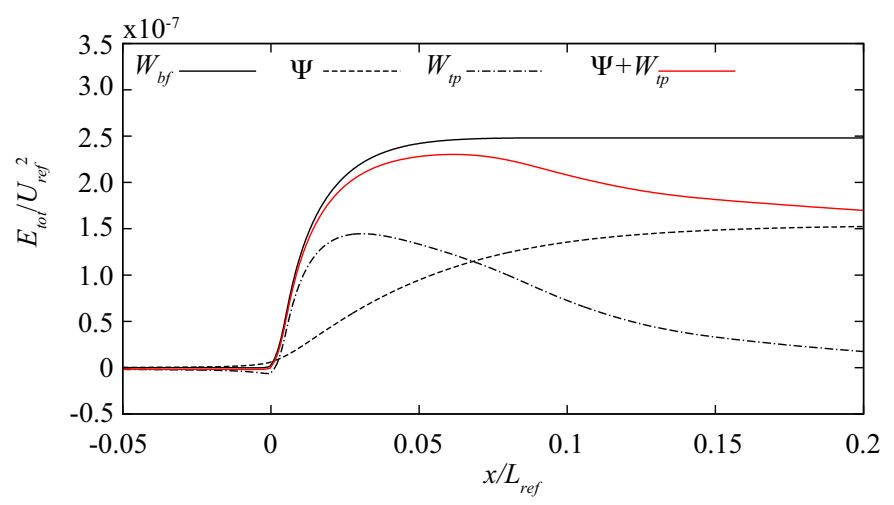

Fig. 22 The energy budget in terms of the work done by the induced velocity and the plasma actuator body force $\left(W_{b f}\right)$, the total pressure $\left(W_{t p}\right)$, and the viscous dissipation function $(\Psi)$. Solid, dashed, and dash-dot lines indicate the body force, the viscous dissipation function, and the total pressure.

\section{References}

Abe, Y., Nonomura, T., Iizuka, N. and Fujii, K., Geometric interpretations and spatial symmetry property of metrics in the conservative form for high-order finite-difference schemes on moving and deforming grids, Journal of Computational Physics, Vol. 260, (2014), pp. 163-203.

Asada, K. and Fujii, K., Computational study of aerodynamic characteristics of an airfoil with DBD plasma actuator, $A J K$ 2011-15027, (2011).

Asada, K., Ninomiya, Y., Oyama, A. and Fujii, K., Airfoil flow experiment on the duty cycle of DBD plasma actuator, AIAA 2009-0531, (2009).

Benard, N. and Moreau, E., Capabilities of the dielectric barrier discharge plasma actuator for multi-frequency excitations, Journal of Physics D: Applied Physics, Vol. 43, (2010), pp. 145201-1-145201-14.

Benard, N. and Moreau, E., Electrical and mechanical characteristics of surface AC dielectric barrier discharge plasma actuators applied to air flow control, Experiment in Fluids, Vol. 55, (2014), 1846.

Cattafesta III, L.,N. and Sheplak, M., Actuators for active flow control, Annual Review of Fluid Mechanics, Vol. 43 (2011), pp. 247-272.

Chakravarthy, S. R., Relaxation methods for unfactored implicit upwind schemes, AIAA-84-0165, (1984). 
Cho, Y-C. and Shyy, W., Adaptive flow control of low-Reynolds number aerodynamics using dielectric barrier discharge actuator, Progress in Aerospace Sciences, Vol. 47, (2010), pp. 495-521.

Corke, T., Post, M. and Orlov, D., Single dielectric barrier discharge plasma enhanced aerodynamics: physics, modeling and applications, Experiments in Fluids, Vol. 46, (2009), pp. 1-26.

Deng, X., Min, Y., Mao, M., Liu, H., Tu, G. and Zhang, H., Further studies on geometric conservation law and applications to high-order finite difference schemes with stationary grids, Journal of Computational Physics, Vol. 239, (2013), pp. 90-111.

Font, G.I., Enloe, C.L. and McLaighlin, T. E., Plasma volumetric effects on the force production of a plasma actuator, AIAA Journal, Vol. 48, (2010), pp. 1869-1874.

Forte, M., Jolibios, J., Moreau, E., Touchard, G., Cazalens, M., Optimization of a dielectric barrier discharge actuator by stationary and non-stationary measurements of the induced flow velocity: application to airflow control, Experiments in Fluids, Vol. 43, (2007), pp. 917-928.

Fujii, K., High-performance computing-based exploration of row control with micro devices, Philosophical Transactions of Royal Society A: Mathematical Physical and Engineering Sciences, Vol. 372 (2014), 20130326.

Fujii, K., Simple ideas for the accuracy and efficiency improvement of the compressible flow simulation methods, Proceedings of the International CFD Workshop on Supersonic Transport Design, (1998).

Gaitonde, D. and Visbal, M.R., Pade type higher-order boundary filters for Navier-Stokes equations, AIAA Journal, Vol. 38, (2000), pp. 2103-2122.

Gaitonde, D., Visbal, M. and Roy, S., A coupled approach for plasma-based flow control simulations of wing sections, AIAA 2006-1205, (2006).

Gaitonde, D., Visbal, M. and Roy, S., Control of flow past a wing section with plasma-based body forces, AIAA 2005 5302, (2005).

Gross, A. and H. F. Fasel., Active flow control for NACA 6-series airfoil at Re=64,200, AIAA Journal, Vol. 48 No. 9 , (2010), pp. 1889-1902.

Ibrahim, I. H. and Skote, M., Boundary condition modifications of the Suzen-Huang plasma actuator model, International Journal of Flow Control, Vol. 3, (2011), pp. 111-130.

Ibrahim, I. H. and Skote, M., Simulations of the linear plasma synthetic jet actuator utilizing a modified Suzan-Huang model, Physics of Fluids, Vol. 24, (2012), 113602.

Kotsonis, M. and Veldhuis, L., Experimental study on dielectric barrier discharge actuators operating in pulse mode, Journal of Applied Physics, Vol. 108, (2010), pp. 113304-1-113304-9.

Lele, S.K., Compact finite difference scheme with spectral-like resolution, Journal of Computational Physics, Vol. 103, (1992), pp.16-22.

Maden, I., Maduta, R., Kriegseis, J., Jakirlic, S., Schwarz, C., Grundmann, S. and C. Tropea, Experimental and computational study of the flow induced by a plasma actuator, International Journal of Heat and Fluid Flow, Vol. 41, (2013), pp. 80-89.

Moreau, E., Airflow control by non-thermal plasma actuators, Journal of Physics D: Applied Physics, Vol. 40, (2007), pp. 605-636.

Nishida, H, Nonomura T. and Abe, T., Three-dimensional simulations of discharge plasma evolution on a dielectric barrier discharge plasma actuator, Journal of Applied Physics, Vol. 115, (2014), 133301.

Nishida, H. and Nonomura, T., ADI-SGS scheme on ideal magnetohydrodynamics, Journal of Computational Physics, Vol. 228, (2009), pp. 3182-3188.

Orlov, D. and Corke, T., Numerical simulation of aerodynamic plasma actuator effects, AIAA 2005-1083, (2005).

Riherd, M. and Roy, S., Serpentine geometry plasma actuators for flow control, Journal of Applied Physics, Vol. 114, (2013), 083303.

Roth, J.R., Sherman, D.M. and Wilkinson, S.P., Boundary layer flow control with a one atmosphere uniform glow discharge plasma, AIAA 98-0328, (1998).

Roth, J.R., Sherman, D.M. and Wilkinson, S.P., Electrohydrodynamic flow control with a glow-discharge surface plasma, AIAA Journal, Vol. 38, (2000), pp. 1166-1172.

Roth, J.R. and X. Dai, Optimization of the aerodynamic plasma actuator as an electrohydrodynamic electrical device, AIAA 2006-1203, (2006).

Sato, M., Aono, H., Yakeno, A., Nonomura, T., Fujii, K., Okada, K. and Asada, K., Multifactorial effects of operating conditions of DBD plasma actuator on laminar-separated-flow control, AIAA Journal (2015), doi: 10.2514/1.J053700. 
Sekimoto, S., Asada, K., Anyoji, M., Nonomura T. and Fujii, K., Comparative study of co-flow and counter blowing DBD plasma actuators for separated flow over an airfoil, AIAA 2012-1137, (2012).

Sekimoto, S., Flow control mechanism of a DBD plasma actuator for airfoils in low speed free-streams, Ph.D dissertation, University of Tokyo, (2015).

Sekimoto, S., Sulaiman, T., Anyoji, M., Nonomura, T. and Fujii, K., Experimental study of nano-second pulse plasma actuator for low Reynolds number flow control, AIAA 2014-0767, (2014).

Shang, J.S. and Huang, P.G, Surface plasma actuators modeling for flow control, Progress in Aerospace Sciences, Vol. 67, (2014), pp. 29-50.

Shyy, W., Jayaraman, B. and Andersson, A., Modeling of glow discharge-induced fluid dynamics, Journal of Applied Physics, Vol. 92, (2002), pp. 6434-6443.

Suzen, Y. B., Huang, P. G., Jacob, J. D. and Ashpis, D. E., Numerical simulations of plasma based flow control application, AIAA 2005-4633, (2005).

Vinokur, M. and Yee, H. C., Extension of efficient low dissipation high-order schemes for 3D curvilinear moving grids, World Scientific, Frontiers of Computational Fluid Dynamics, (2002), pp. 129-164.

Visbal, M., Strategies for control of transitional and turbulent flows using plasma-based actuators, International Journal of Computational Fluid Dynamics, Vol. 7, (2010), pp. 237-258.

Wang, J.-J., Choi, K-S., Feng, L-H., Jukes, T. and Whalley, R. D., Recent developments in DBD plasma flow control, Progress in Aerospace Sciences, Vol. 63, (2013), pp. 52-78.

Yamamoto, S. and Fukagata, K., Numerical simulation of a plasma actuator based on ion transport, Journal of Applied Physics, Vol. 113, (2013), 243302. 